\title{
Agregación costera de tortugas marinas en aguas del Pacífico Sur de Costa Rica
}

\author{
Rubén Venegas-Li ${ }^{1}$, José David Palacios Alfaro ${ }^{2} \&$ Damián Martínez Fernández ${ }^{3}$ \\ 1. ARC Centre of Excellence for Environmental Decisions, School of Biological Sciences, The University of \\ Queensland, Brisbane 4072, Australia; r.venegas@uq.edu.au \\ 2. Fundación Keto, San José, Costa Rica; dpalacios@fundacionketo.org \\ 3. Proyecto Consolidación de Áreas Marinas Protegidas de Costa Rica SINAC-PNUD-GEF, San José, Costa Rica; \\ damian.martinez@sinac.go.cr
}

Recibido 11-VIII-2014. Corregido 20-XI-2014. Aceptado 22-XII-2014.

\begin{abstract}
Coastal aggregation of marine turtles in waters of the South Pacific of Costa Rica. The southern Pacific coast of Costa Rica has been identified as an important place for the reproduction of four species of marine turtles. This presence, coupled with threats to the survival of the species, has encouraged the establishment of conservation initiatives and the study of these animals in the region, especially in nesting areas. Nonetheless, information on their presence in the water (where they spend most of their life) is scarce. For this reason, this study analyses data from a three years of a marine turtle monitoring program by Fundación Keto in the area between the Marino Ballena National Park and the Caño Island Biological Reserve, and presents the temporal and spatial distribution of the observed individuals. During this period, a total of 447 turtle (sightings per sampling effort $=0.36$ individuals $-\mathrm{hr}^{-1}$ ), of three different species were observed: Lepidochelys olivacea, Chelonia Mydas, and Eretmochelys imbricata. Sightings have been recorded during all months of the year, with L. olivacea as the species most commonly observed, especially outside of protected areas; this species presented a clear mating period in the months of July and August. E. imbricata was the species observed closer to the shore, and at a shallower site, possibly indicating an important foraging area for juvenile individuals. This is the first study to address the consistent and permanent presence of three species of marine turtles in the waters off the coast of Cantón de Osa in the southern Pacific coast of Costa Rica. Rev. Biol. Trop. 63 (Suppl. 1): 1-8. Epub 2015 April 01.
\end{abstract}

Key words: Marine turtles, Osa, Lepidochelys olivacea, Chelonia mydas, Eretmochelys imbricata, distribution, Marino Ballena National Park.

Las costas del Pacifico Sur de Costa Rica son importantes para la reproducción de cuatro especies de tortugas marinas, con al menos nueve playas de anidación en el cantón de Osa, Puntarenas (Chacón, Sánchez, Calvo \& Ash, 2007). Estas cuatro especies son: la tortuga lora (Lepidochelys olivacea [Eschscholtz, 1829]), la tortuga verde (Chelonia mydas [Linnaeus 1758]), la carey (Eretmochelys imbricata [Linnaeus 1766]), y la tortuga baula (Dermochelys coriacea [Vandelli, 1761]).

Estas nueve playas de anidación han sido monitoreadas los últimos años para determinar el comportamiento de anidación de las tortugas marinas (Sánchez, 2006, 2007; Retana, 2008;
Brenes-Arias, 2009, 2010; Villareal, 2011; James, 2011). Mediante este monitoreo, se ha obtenido información sobre parte del ciclo reproductivo de sus poblaciones (p.e. fechas de anidación, número de nidadas, esfuerzo reproductivo intra e interanual), y se han identificado las amenazas que enfrentan (p.e. saqueo y depredación de nidos, matanza por su carne y caparazón, pesca incidental).

Las tortugas marinas pasan su vida esencialmente en hábitats marinos o estuarinos, utilizando el hábitat terrestre solamente para la anidación y en casos restringidos para calentarse al sol (Musick \& Limpus, 1997). Por lo anterior, es fundamental recopilar información 
sobre su distribución, abundancia y comportamiento en las zonas pelágicas y neríticas, de manera que se complemente la información obtenida en playas de anidación, que permita una formulación de estrategias de conservación más efectivas (Wallace et al., 2013). Sin embargo, el elevado costo de monitorear zonas marinas hace que este tipo de estudios sean escasos (Seminoff, Jones, Resendiz, Nichols \& Chaloupka, 2003), y las costas del Pacífico Sur de Costa Rica no son una excepción.

Por lo anterior, el objetivo de este estudio es determinar espacial y temporalmente las zonas de agregación de tortugas marinas, con énfasis en el Parque Nacional Marino Ballena, la Reserva Biológica Isla del Caño y sus alrededores. Se espera que esta información contribuya para el diseño de estrategias de conservación efectivas tanto de las tortugas marinas como de los sitios que habitan.

\section{MATERIALES Y MÉTODOS}

Área de estudio: Los muestreos se realizaron entre la zona frente a playa Dominicalito (913'12,1" N - 83 $52^{\circ}$ '5,3" W) en el extremo norte del cantón de Osa en el Pacífico Sur de Costa Rica, y la Isla del Caño (8³9'31.7” N $83^{\circ} 56^{\prime} 4.1^{\prime \prime} \mathrm{W}$ ), a una distancia máxima aproximada de la costa de $33 \mathrm{~km}$. El área de la zona de muestreo abarca aproximadamente $1280 \mathrm{~km}^{2}$, e incluye las áreas marinas protegidas del Parque Nacional Marino Ballena (PNMB) y de la Reserva Biológica Isla del Caño (RBIC).

Registro de datos: La información fue recolectada desde plataformas de oportunidad, en este caso botes de turismo para avistamiento de cetáceos que operan desde Bahía Ballena y Bahía Drake. Los muestreos se realizaron entre enero de 2009 y diciembre de 2011, de las 08:00 a las $16 \mathrm{~h}$ siguiendo rutas establecidas por los capitanes de las embarcaciones, entre las áreas marinas protegidas y paralelas a la costa. En cada avistamiento se registró la posición geográfica, fecha, hora, especie, número de individuos, estado (vivo o muerto) y comportamiento (apareamiento o no apareamiento).
El tiempo máximo de observación no excedió nunca los 3 minutos aproximadamente, ya que la observación de las tortugas era un evento incidental y no el objetivo de los viajes.

Análisis de datos: El número de avistamientos se estandarizó utilizando valores de avistamiento por unidad de esfuerzo (APUE), expresado como el número de individuos avistados por hora de esfuerzo al día (individuos-hr-1). Para determinar diferencias en la distribución espacial de las especies observadas, se aplicó un Análisis de Varianza (ANOVA) relacionando las profundidades (en metros, m) a la que se observó cada individuo, así como su distancia a la costa.

\section{RESULTADOS}

Se realizó un esfuerzo de muestreo total de $1253.13 \mathrm{~h}$ registradas en 425 salidas, realizadas en 360 días de monitoreo. El esfuerzo promedio por cada salida fue de $2.95 \pm 1.22 \mathrm{~h}$. Se debe mencionar que en el caso específico del año 2011, en los meses de abril, mayo, junio, noviembre y diciembre, el esfuerzo de muestreo fue igual a cero.

En total, se avistaron 447 tortugas marinas, de las cuales 384 fueron identificadas positivamente a nivel de especie, y correspondieron a L. olivacea $(\mathrm{n}=292)$, E. imbricata $(\mathrm{n}=63)$, y $C$. mydas $(\mathrm{n}=25)$, mientras que 63 individuos no fueron identificadas (por dificultad de visibilidad) siendo agrupadas en "tortugas no identificadas".

La Fig. 1 muestra la distribución de los sitios donde ocurrieron los avistamientos de tortugas marinas. El 50\% de los avistamientos fueron realizados a una distancia de la costa entre $1.94 \mathrm{~km}$ y $12.48 \mathrm{~km}$ (mediana $=5.89 \mathrm{~km}$ ), y a una distancia mínima de $0.01 \mathrm{~km}$ y máxima de $51.3 \mathrm{~km}$. Asimismo, el $50 \%$ de las tortugas observadas estaban a profundidades entre $27.21 \mathrm{~m}$ y $73.94 \mathrm{~m}$ (mediana $=56.5$ ). Las observaciones a una distancia mayor a los $25 \mathrm{~km}$ de la costa $(n=11)$, y en los que las profundidades eran mayores a $1500 \mathrm{~m}$ fueron realizados en una sola ocasión (un viaje con fines científicos); 


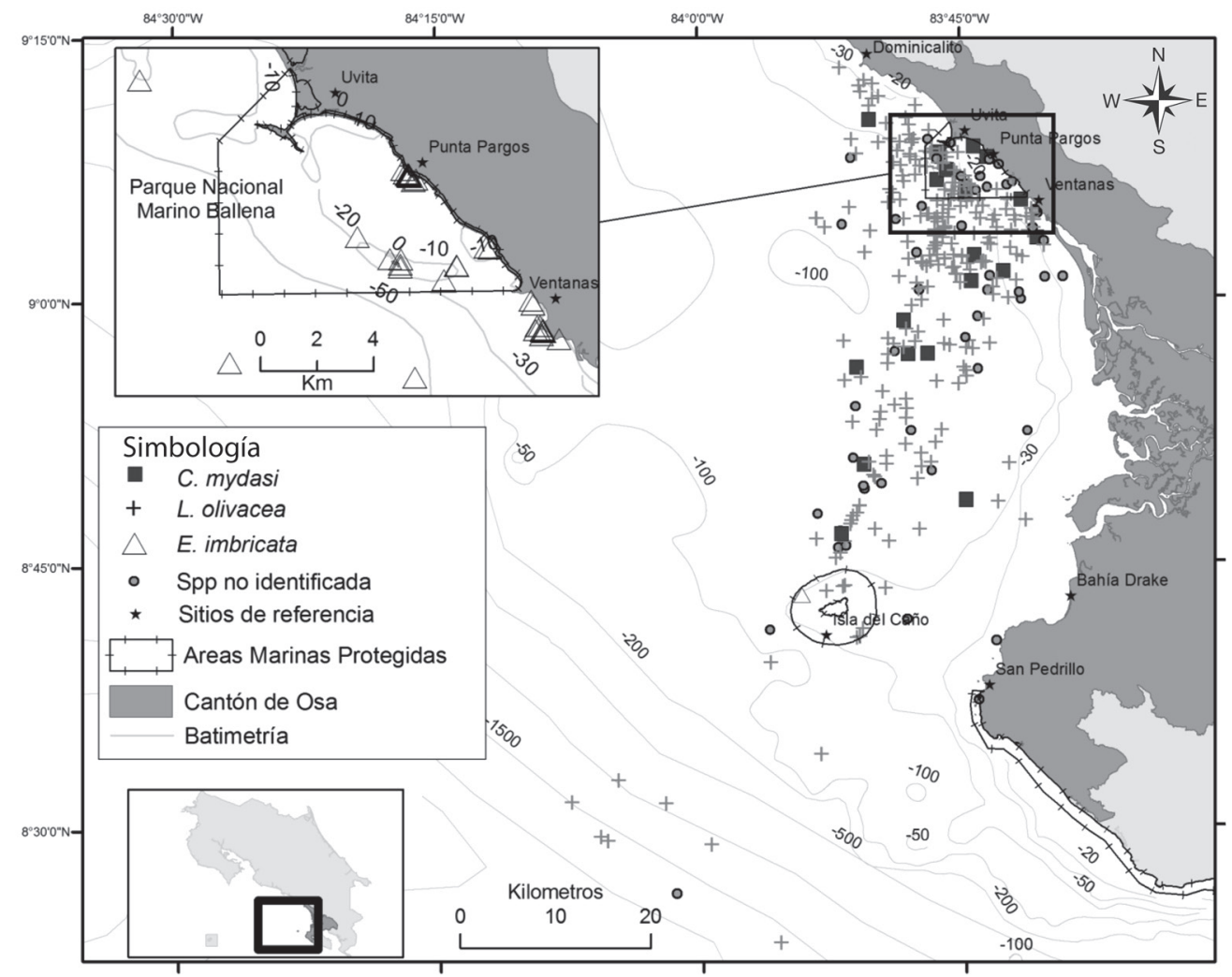

Fig. 1. Avistamientos de tortugas marinas en el Pacífico Sur de Costa Rica, entre los años 2009-2011. En el recuadro de la esquina superior izquierda, sólo se muestran los avistamientos de E. imbricata.

Fig. 1. Sightings of marine turtle in the South Pacific of Costa Rica, years 2009-2011. The inset at the upper left corner shows only sightings of E. imbricata.

todos los demás avistamientos fueron hechos en zonas en las que la profundidad era menor a los $100 \mathrm{~m}$.

Como se observa en la Fig. 2, E. imbricata ocupó zonas más cercanas a la costa $(\mathrm{F}=10.6$, g.l. $=3, \quad \mathrm{p}<0.01)$ y a profundidades menores $(\mathrm{F}=2.4$, g.1. $=3, \mathrm{p}<0.5)$ que el resto de las especies. Además, sólo 12 individuos de L. olivacea fueron avistados a profundidades mayores a $100 \mathrm{~m}$, de los cuales 11 se registraron en una misma salida (7 de febrero del 2009).

El APUE total fue de 0.36 individuos-hr ${ }^{-1}$. L. olivacea fue la especie más avistada $(0.23$ individuos-hr-1 $\left.{ }^{-1}\right)$, seguido por E. imbricata $(0.05$ individuos-hr-1), y C. mydas (0.02 individuos$\mathrm{hr}^{-1}$, Fig. 3). Para L. olivacea el APUE mensual considerablemente más alto se obtuvo durante julio y agosto (Fig. 3), y nulo en noviembre y diciembre. E. imbricata presentó el máximo APUE en mayo $\left(0,1\right.$ individuos-hr- $\left.{ }^{-1}\right)$, mientras que C. mydas, en enero de 2011 (0,6 individuos-hr- ${ }^{-1}$ ).

Se debe mencionar que en 47 eventos se observó comportamiento de apareamiento de tortugas marinas. De éstos, 33 fueron de $L$. olivacea, lo cual indica que al menos en $13.5 \%$ de los avistamientos esta especie estaba en fase de reproducción; la mayoría de estos eventos (28) se observaron entre los meses de julio y agosto. Además, se registraron 11 eventos de apareamiento de C. mydas, que representa el $55 \%$ de los avistamientos de esta especie.

Finalmente, se observaron diez individuos de L. olivacea muertos, de los cuales sólo para uno se pudo determinar la causa de la muerte (golpe por un bote de turismo). Los 


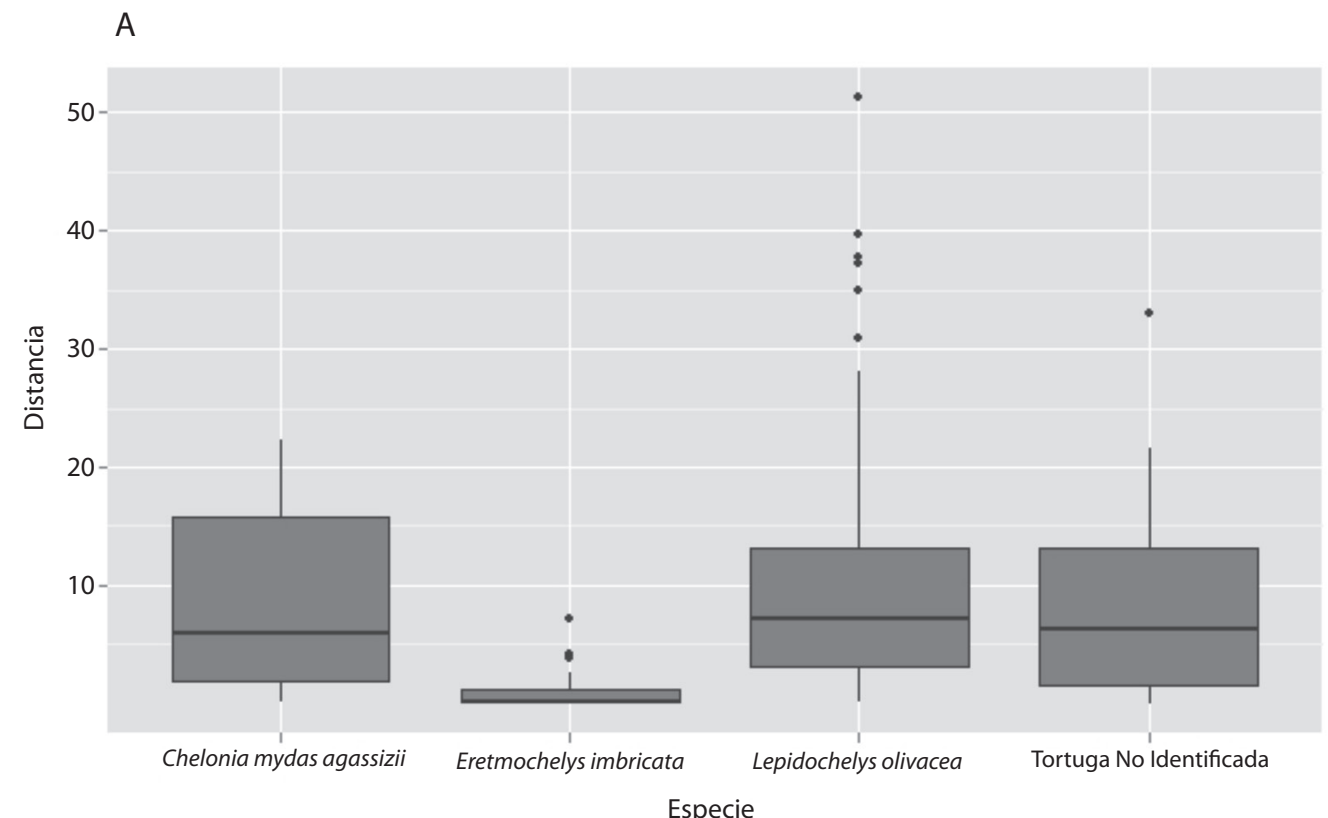

B

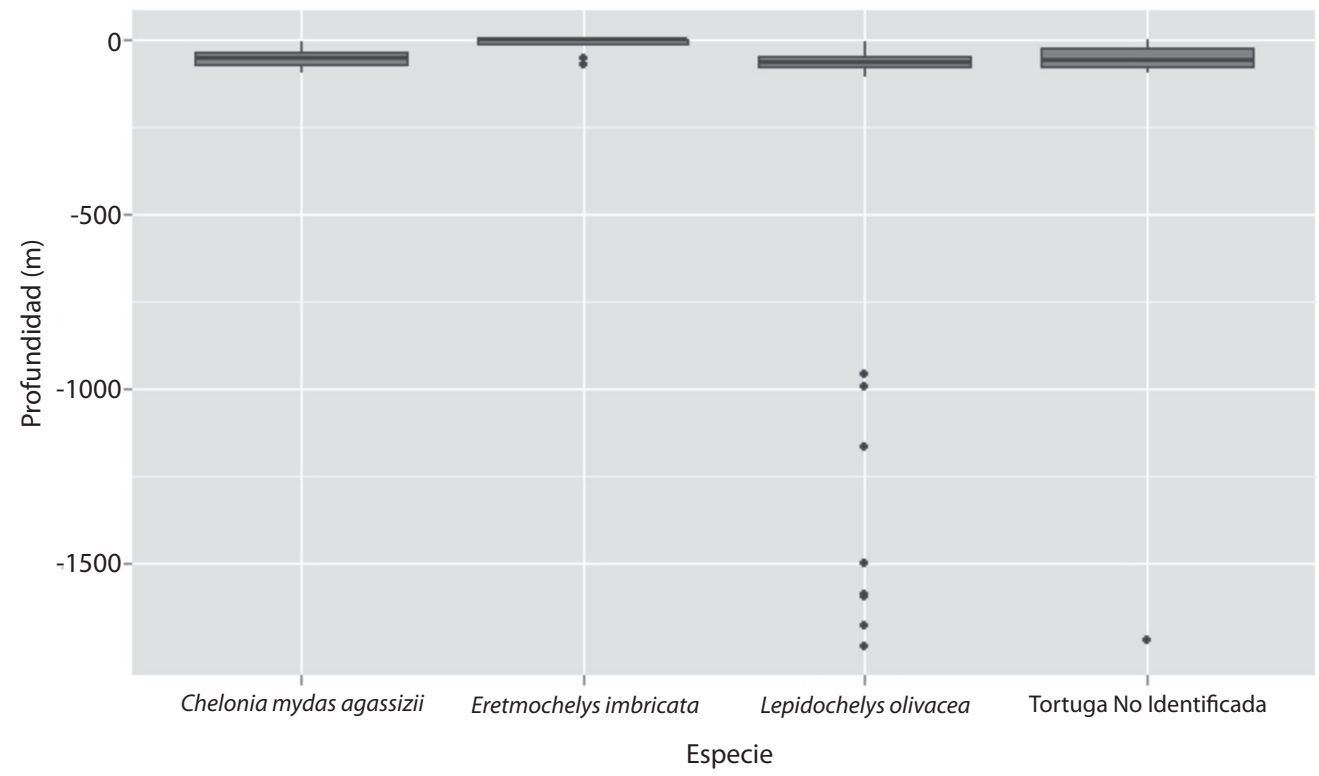

Fig. 2. Distancia a la costa (A) y profundidad (B) de los avistamientos de tres especies de tortugas marinas en el Pacífico Sur de Costa Rica, entre los años 2009-2011.

Fig. 2. Distance to the coast (A) and depth (B) of the sightings of three species of marine turtles in the South Pacific of Costa Rica, years 2009-2011. 


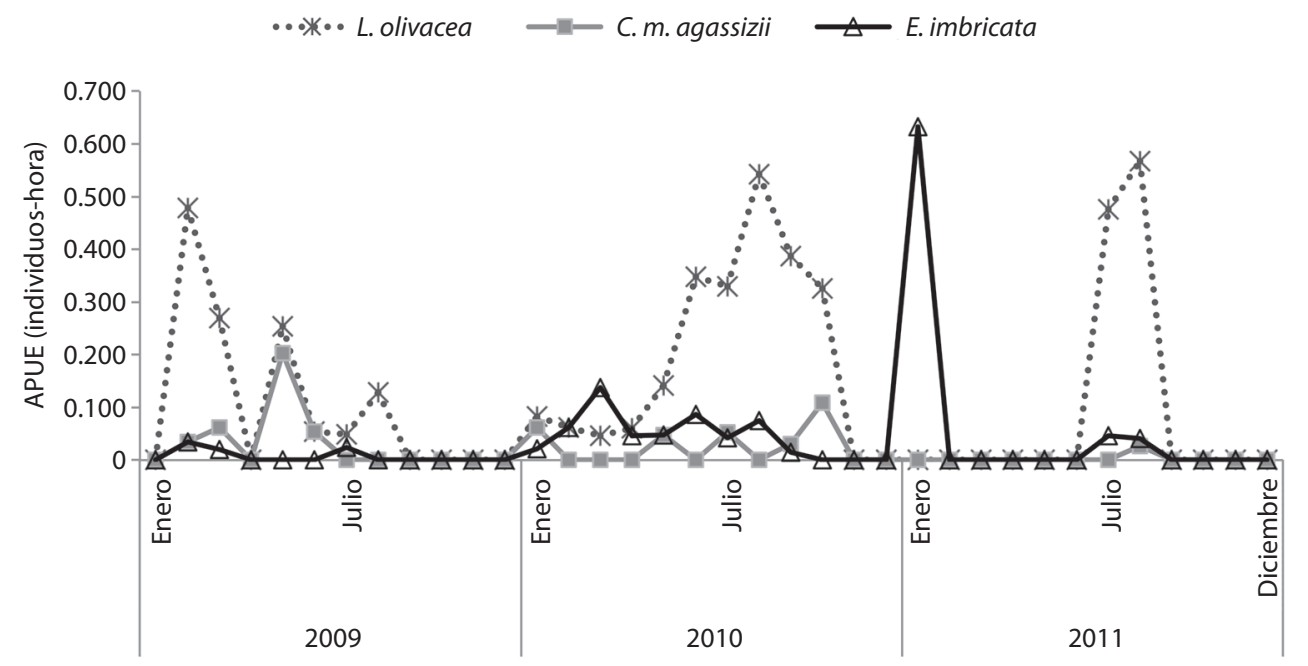

Fig. 3. Avistamiento por unidad de esfuerzo de tres especies de tortugas marinas entre los años 2009 y 2011 en las aguas frente a las costas del cantón de Osa, Pacífico Sur de Costa Rica.

Fig. 3. Sightings per unit of effort of three species of marine turtles between 2009 and 2011 in the water in front of the coast of Cantón de Osa, South Pacific of Costa Rica.

demás ejemplares presentaron avanzado estado de descomposición.

\section{DISCUSIÓN}

Este es el primer aporte científico que documenta la presencia exacta, considerable y continua a lo largo del año de L. olivacea, E. imbricata y $C$. mydas en aguas frente a las costas del cantón de Osa, en el Pacífico Sur de Costa Rica. Si bien Morant-Sánchez, Goodman, Sancho \& Julsing (2004) reportaron avistamientos de tortugas entre 2001 y 2004 en salidas de turismo al mar, estos reportes se limitaron a L. olivacea, sin dar a conocer la ubicación exacta de los avistamientos. Por otra parte, Bessesen (2011) reportó avistamientos de cuatro especies de tortugas en aguas del Golfo Dulce.

Las especies observadas concuerdan con tres de las cuatro especies de tortugas marinas reportadas en el estudio mencionado anteriormente por Bessesen (2011). Esto indica que la zona evaluada no es única en presencia de tortugas marinas en el Pacífico Sur de Costa Rica, lo cual debe considerarse al diseñar planes de manejo integral de las poblaciones de tortugas marinas, pues los individuos podrían moverse entre una zona y otra.

La ausencia de avistamientos de tortuga Baula (D. coriacea) puede deberse a los bajos esfuerzos de muestreo en los meses de noviembre y diciembre -los más importantes para la anidación de esta especie-, y a los bajos números de anidación de esta especie en la zona (Govan, 1998). Es interesante observar además, que un estudio (Shillinger et al., 2011) de movimientos post-anidatorios de hembras que anidan en Playa Grande, no registró movimientos importantes a esta zona.

Lepidochelys olivacea fue la especie más observada, lo que coincide con el hecho de ser la especie con mayor números de anidamientos en la zona (Drake, 1996; Barquero-Edge, 2013), y para la cual Govan y Adecoro (1996) estimaron mil hembras anidantes en una temporada de anidación. Además, los meses con mayor APUE y avistamientos de parejas copulando (julio y agosto) coinciden con el inicio de la época de mayor anidación en las playas del cantón de Osa (Drake, 1996; Govan, 1998; Sánchez, 2006, 2007; BrenesArias, 2009, 2010; Barquero-Edge, 2013). Esto señala la importancia de la zona de estudio para 
la reproducción de L. olivacea, puesto no sólo se registra la presencia de ejemplares hembras sino también de machos.

En este estudio se incluyen datos del único muestreo realizado a profundidades mayores a los $1000 \mathrm{~m}$, registrándose 11 avistamientos de de tortuga lora, lo que indica que estos individuos no están restringidos a zonas someras, y demuestra la importancia de extender el esfuerzo de muestreo a zonas alejadas de la costa. Pitman (1990) reporta que esta especie es la más abundante en el Tropical Oriental, y que puede ser observada a más de $1850 \mathrm{~km}$ en aguas oceánicas lejos de la costa, prácticamente todos los meses.

Los avistamientos de E. imbricata en aguas poco profundas y muy cercanas a la costa, especialmente frente a Playa Ventanas y Punta Pargos, indican que estos lugares son importantes zonas de alimentación para la especie, y respalda el reporte de estos sitios como potenciales zonas de alimentación para la especie (Widecast, 2011). Estos individuos podrían estar haciendo un uso de estos hábitats similar al descrito por Carrión-Cortez, CanalesCerro, Arauz y Riosmena-Rodríguez (2013), quien describe la dieta y los patrones de movimientos para la población de tortuga carey en Punta Coyote, Pacífico Norte de Costa Rica. Por otro lado, a pesar de la baja presencia de tortugas carey observadas en los alrededores de la isla del Caño desde las embarcaciones, estas son observadas en los sitios de esnorkel con cierta frecuencia (D. Palacios, en prep.).

La mayoría de los avistamientos ocurrió fuera del PNMB y de la RBIC, lo cual demuestra que existe una extensa área donde estos animales pueden ser más vulnerables a amenazas antrópicas como por ejemplo golpes por botes y pesca incidental.

$\mathrm{Al}$ respecto, amenazas como la pesca incidental de estos organismos ha sido registrada anteriormente en la zona de estudio (BIOMARCC-SINAC-GIZ 2012). Para entender mejor la potencial seriedad de la pesca incidental de tortugas marinas en la zona de estudio, se puede observar registros anteriores en aguas costarricenses como los reportados por Arauz,
Vargas, Naranjo y Gamboa (1998) y Whoriskey, Arauz y Baum (2011), los cuales estiman capturas de hasta 15631 individuos en pesca de arrastre y de 14 individuos por cada 1000 anzuelos en la pesca de palangre, respectivamente.

Los resultados obtenidos contribuyen a generar información sobre un grupo taxonómico identificado como objeto de conservación en la elaboración de GRUAS II, plan de ordenamiento territorial para Costa Rica en el que se establecieron prioridades de conservación, por lo que el conocimiento generado debería incorporarse en la toma de decisiones de manejo en la zona de estudio.

No obstante, quedan muchas preguntas sin responder en cuanto a la presencia y comportamiento de las tortugas marinas en la zona de estudio (y fuera de esta), por lo que se recomienda la ejecución de estudios que permitan conocer si los individuos observados son residentes o no, o cuánto tiempo pasan en la zona (si son residentes o son residentes temporales), cuál es el tamaño de sus poblaciones, la proporción de sexos y el uso que hacen del hábitat (p.e. zona de alimentación), entre otros aspectos.

\section{CONCLUSIONES}

Se demuestra la presencia de tortugas marinas (hembras y machos) en el área de estudio, desde aguas costeras hasta más de $30 \mathrm{~km}$ de la costa; sin embargo, se recomienda realizar muestreos sistemáticos para cubrir una mayor zona.

Es importante destacar los resultados que surgen a partir de alianzas entre ONGs y la empresa privada, en donde la utilización de embarcaciones turísticas representa una forma accesible y de bajo costo que permite obtener información básica (distribución, número de individuos, etc.) de especies de tortugas marinas en peligro de extinción o con poblaciones reducidas en el país. Se recomienda complementar este tipo de muestreo con embarcaciones exclusivas de investigación y además valorar factores que pueden afectar la detectabilidad de las tortugas marinas en el mar, 
como lo son el oleaje, el viento, velocidad de la embarcación y patrones de buceo, entre otros.

\section{AGRADECIMIENTOS}

Se agradece la colaboración en campo de la Asociación de Tour Operadores de Uvita, a las tour operadoras Bahía Aventuras, Ballena Aventura, Bahía Ballena Tour, Dolphin Tour, Ballena Tour, La Colonia Tour, Fragata Tour, Gurrión Tour, Kefersi Tour, Pacific Whales and Kayak tour, Pelican tour y Winsheale Tour. A Julio y Marlene Badilla, Mauricio Quesada, Ronald Monge, Paula Ramírez, Ronald Monge, Fernando Monge, Endre y Alejandro Vásquez, José Mesen, Rafael Ortega, Óscar, Fernando Guerrero, Charlie León, Luz Miriam Badilla, Walter Brenes, Reymer Brenes, Rafael Rosales, Junior Monge, Carlos Salas, Jovino Vargas, Antonio Quesada, Roy Quesada, Eduardo Quesada, Alejandro Orozco, Dennis Mora. A Tomas González, Claudia Matzdorf, Sonya Gallego, Maite Urrutxua, Melanie y Álvaro Sauma por su colaboración en la recolección de información. Al Sistema Nacional de Áreas de Conservación, especialmente al Área de Conservación Osa por su apoyo logístico. Finalmente, a dos revisores anónimos del manuscrito por sus valiosas sugerencias.

\section{RESUMEN}

El Pacífico Sur de Costa Rica ha sido identificado como un sitio importante para la reproducción de cuatro especies de tortugas marinas. Las amenazas que éstas sufren han originado iniciativas de conservación y estudio en la zona, especialmente en playas de anidación. Sin embargo, información sobre su presencia en el agua (donde pasan la mayor parte de su vida) es escasa. Por esta razón, este estudio analiza la información recolectada como parte de un proyecto de monitoreo de tortugas marinas de la Fundación Keto, en la zona comprendida entre el Parque Nacional Marino Ballena y la Reserva Biológica Isla del Caño. Entre los años 2009 y 2011, se registraron 447 avistamientos de tortugas (la mayoría fuera de las áreas de conservación) pertenecientes a tres especies: Lepidochelys olivacea, Chelonia mydas, y Eretmochelys imbricata. Se registraron avistamientos durante todos los meses del año, siendo L. olivacea la especie con más individuos observados. Se identificaron dos áreas (a profundidades menores a $10 \mathrm{~m}$ ) importantes para la presencia de E. imbricata. Este es el primer aporte científico que documenta la presencia exacta, considerable y continua en el tiempo de tres especies de tortugas marinas frente a las costas del Cantón de Osa, Puntarenas, Costa Rica.

Palabras clave: Tortugas marinas, Osa, Lepidochelys olivacea, Chelonia mydas, Eretmochelys imbricata, distribución, Parque Nacional Marino Ballena.

\section{REFERENCIAS}

Arauz, R. M., Vargas, R., Naranjo, I., \& Gamboa, C. (1998). Analysis of the incidental capture and mortality of sea turtles in the shrimp fleet of Pacific Costa Rica. In S. P. Epperly \& J. Braun (Eds.), Proceedings of the seventeenth annual sea turtle symposium (pp. 1-5). USA: NOAA Techical Memorandum NMFS-SEFSC-415.

Barquero-Edge, P. (2013). Trends in Marine Turtle Nesting and Egg Predation on the Osa Peninsula, Costa Rica. Marine Turtle Newsletter, 138, 7-10.

Bessesen, B. L. (2011). Rainy season extension of the multi-species marine sighting survey in Golfo Dulce, Costa Rica, July-August 2011. San José, Costa Rica.

BIOMARCC, SINAC, \& GIZ. (2012). Estudios cientificos de hábitat marino costero y situación socioeconómica del Pacífico Sur de Costa Rica. San José, Costa Rica.

Brenes-Arias, O. (2009). Proyecto de conservación de Tortugas Marinas, Playa Tortuga, Ojochal de Osa, Puntarenas, Costa Rica, Temporada 2009. San José, Costa Rica.

Brenes-Arias, O. (2010). Proyecto de conservación de Tortugas Marinas, Playa Tortuga, Ojochal de Osa, Puntarenas, Costa Rica, Temporada 2010. San José, Costa Rica.

Carrión-Cortez, J., Canales-Cerro, C., Arauz, R., \& Riosmena-Rodríguez, R. (2013). Habitat Use and Diet of Juvenile Eastern Pacific Hawksbill Turtles (Eretmochelys imbricata) in the North Pacific Coast of Costa Rica. Chelonian Conservation and Biology, 12(2), 235-245.

Chacón, D., Sánchez, J., Calvo, J. J., \& Ash, J. (2007). Manual para el manejo y la conservación de las tortugas marinas en Costa Rica; con énfasis en la operación de proyectos en playa y viveros. Costa Rica: Sistema Nacional de Áreas de Conservación, Ministerio de Ambiente y Energía.

Drake, D. (1996). Marine Turtle Nesting, Nest Predation, Hatch Frecuency and Nesting Seasonality on the Osa Peninsula, Costa Rica. Chelonian Conservation and Biology, 2(1), 89-92.

Govan, H., \& ADECORO. (1996). El recurso de tortugas marinas en río Oro, Península de Osa. San José, Costa Rica. 
Govan, H. (1998). Community turtle conservation at Río Oro on the Pacific coast of Costa Rica. Marine Turtle Newsletter, 80, 10-11.

James, R. (2011). Investigation and Conservation of Sea Turtles Drake Bay, Osa Peninsula, Costa Rica, Technical Report. San José, Costa Rica.

Morant-Sanchez, C., Goodman, S., Sancho, R., \& Julsing, S. (2004). Turtle Report, Fundación Vida Marina. San José, Costa Rica.

Musick, J. A., \& Limpus, C. J. (1997). Reproduction in sea turtles. In P. Lutz \& J. A. Musick (Eds.), The Biology of Sea Turtles Volume I (pp. 137-163). USA: CRC Press LLC.

Pitman, R. L. (1990). Distribution and biology of sea turtles in the eastern tropical Pacific. In T. H. Richardson, J. I. Richardson \& M. Donelly (Eds.), Proceedings of the Tenth Annual Workshop on Sea Turtle Conservation and Biology (pp. 143-149). USA: NOAA Techical Memorandum NMFS-SEFC-278.

Retana, G. (2008). Reporte de Temporada 2007. Programa Conservación de Tortugas Marinas Playa Piro. San José, Costa Rica.

Sánchez, F. (2006). Programa de conservación de tortugas marinas de la Península de Osa, Playas Carate, Río Oro, Pejeperro Y Piro(Reporte técnico temporada 2005). PRETOMA: Costa Rica.

Sánchez, F. (2007). Programa de conservación, investigación y educación de tortugas marinas en la Península de Osa, Playas Carate, Río Oro, Pejeperro y Piro (Reporte técnico temporada 2006). Corcovado Fundation: Costa Rica.

Seminoff, J. A., Jones, T. T., Resendiz, A., Nichols, W. J., \& Chaloupka, M. Y. (2003). Monitoring green turtles (Chelonia mydas) at a coastal foraging area in Baja California, Mexico: multiple indices to describe population status. Journal of the Marine Biological Association of the United Kingdom, 83(6), 1355-1362

Shillinger, G., Swithenbank, A., Bailey, H., Bograd, S., Castelton, M., Wallace, B., ... Block, B. (2011). Vertical and horizontal habitat preferences of post-nesting leatherback turtles in the South Pacific Ocean. Marine Ecology Progress Series, 422, 275-289.

Villareal, A. (2011). Informe Técnico de Temporada 20102011, RNVS Hacienda Barú. San José, Costa Rica.

Wallace, B. P., Kot, C. Y., DiMatteo, A. D., Lee, T., Crowder, L. B., \& Lewison, R. L. (2013). Impacts of fisheries bycatch on marine turtle populations worldwide: toward conservation and research priorities. Ecosphere, 4(3), 40.

WIDECAST. (2011). Report of Activities Pacific Hawksbill Sea Turtles Program in Osa region, Costa Rica. San José, Costa Rica.

Whoriskey, S., Arauz, R., \& Baum, J. K. (2011). Potential impacts of emerging mahi-mahi fisheries on sea turtle and elasmobranch bycatch species. Biological Conservation, 144(6), 1841-1849. 